\title{
Physical Properties of Ophthalmic Copolymers Containing Vinylaniline and ZnO Nanoparticles"
}

\author{
A-Young Sung ${ }^{1 \#}$, Tae-Hun Kim ${ }^{2}$ \\ ${ }^{1}$ Department of Ophthalmic Optics, Sehan University, Jeonnam, South Korea; ${ }^{2}$ Department of Visual Optics, Baekseok University, \\ Chonan, South Korea. \\ Email: " say123sg@hanmail.net
}

Received May 25 ${ }^{\text {th }}, 2013$; revised June 26 ${ }^{\text {th }}, 2013$; accepted July $15^{\text {th }}, 2013$

Copyright (C 2013 A-Young Sung, Tae-Hun Kim. This is an open access article distributed under the Creative Commons Attribution License, which permits unrestricted use, distribution, and reproduction in any medium, provided the original work is properly cited.

\begin{abstract}
The mixture of vinylaniline and 2-hydroxyethyl mrthacrylate mixed according to the mixing ratio, was allowed to stir in order to add functionality. $\mathrm{ZnO}$ nanoparticles, dispersed for approximately $1 \mathrm{hr}$ in an ultrasonic bench top cleaner, were added to the mixture of monomers. Then the mixture at a mould was thermally polymerized to prepare high-performance hydrogel ophthalmic lens. The produced ophthalmic hydrogel lens was then put in $0.9 \% \mathrm{NaCl}$ (normal saline solution) for 24 hrs for hydration, and various measurements were carried out. The surface analysis of ZnO nanoparticles dispersed in the macromolecular polymer was conducted using AFM (XE-100, Parks System). The produced hydrogel lens generally exhibited yellowish color, transparent and hydrophilic characteristics. Also, the contact lenses hydrated in standard saline solution generally exhibited the characteristics of flexibility and softness. The physical properties of the ophthalmic hydrogel lens material were measured. The refractive indexes of $1.359-1.423$, water content of $37 \%-43 \%$, and visible transmittance of $74 \%$ - $82 \%$ were obtained.
\end{abstract}

Keywords: ZnO Nanoparticles; Vinylaniline; Spectral Transmittances; Contact Angles

\section{Introduction}

Recently, there has been active research on not only basic properties such as water content, wettability and tensile strength, but also on functional properties such as high oxygen permeability, antimicrobial quality and UV protection, etc., in the study of contact lens materials [1-6]. Research on the antimicrobial quality of contact lens materials is booming, with an emphasis on nanoparticles that are very widely applied in the industrial and medicine areas [7-9]. As nano-composite materials have unique electrical, magnetic, optical and engineering applicability due to their size-effect and quantum confinement effect, they are being extensively studied in various fields. Currently, research of applying nano-composite material to contact lenses is placing a disproportionate emphasis on how to apply nano-silver and nano-gold for antimicrobial quality. The optical features of nano-composites, however, are one of the crucial characteristics

\footnotetext{
${ }^{*}$ This research was financially supported by Ministry of Trade, Industry \& Energy (MOTIE) and Korea Evaluation Institute of Industrial Technology (KEIT) through the Project of Standard Technology Development.

${ }^{\#}$ Corresponding author.
}

that have recently been brought into spotlight. The ultraviolet blocking ability of contact lenses is recently in the center of attention and is one of the very important optical properties. Ultraviolet rays can result in damage to the cornea, the retina and the eye lens. Also, the damage to the eye lens may cause cataracts and photic maculopathy [10]. In regards to this, Duker-Elder [11], Zigman [12] and others revealed their research results through animal tests under UV-B 280 - $320 \mathrm{~nm}$, which demonstrated that ultraviolet rays could cause eye lens opacity. Various types of damage to cornea as well as the eye lens can lead to degeneration. And if the damage is acute, it can lead to keratitis [13-15]. Therefore, the significance of ultraviolet reduction through contact lenses is becoming more and more important. Research on ultraviolet blocking contact lenses revolved around benzophenone-group substances, but recently it has been extended to other materials such as vinyl pyridine and vinylanisole [16,17]. In addition, there has been research on contact lens materials that are able to block not only ultraviolet rays, but also infrared rays by using nanocomposites such as silicon 2,3-naphthalocyanine bis(trihexylsilyloxide) and silicon 2,9,16,23-tetra-tert-butyl- 
29H31H-phthalocyanine dihydroxyde [18].

$\mathrm{ZnO}$ nanoparticles have a strong luminescence property in the ultraviolet region due to the broadband gap energy and high excitation binding energy at room temperature. Hence, it is used for semiconducting, piezoelectric, photoconducting, and other applications and is widely applied to display screens using optical properties, the electrodes of photocells, light-emitting diodes, laser diodes, photovoltaic devices, etc. For that reason, while $\mathrm{ZnO}$ nanoparticles are regarded as an important material for the future, it has not yet been applied as a contact lens material.

In this research, contact lenses were polymerized by using $\mathrm{ZnO}$ nanoparticles. The physical and optical characteristics of these lenses were then examined. If a pale pigment is added to a contact lens material, it helps to distinguish the lenses better than transparent ones and can be used for a wider variety of applications. Therefore, 4-vinylaniline, which is used in the dye composition, was added to mixture as an additive to analyze its properties.

\section{Experiments and Methods}

\subsection{Reagents and Materials}

HEMA (2-hydroxyethyl methacrylate), which was the main material of hydrophilic hydrogel lenses used in the test, and AIBN (azobisisobutyronitrile), an initiator used for polymerization, were purchased from JUNSEI. NVP (n-vinylpyrrolidone) and EGDMA (ethylene glycol dimethacrylate) were purchased from Acros whereas MMA (methyl methacrylate) was purchased from Crown Guaranteed Reagents. 4-Vinylaniline and $\mathrm{ZnO}$ (nanopowder, $100 \mathrm{~nm}$ particle size) were purchased from Aldrich.

\subsection{Polymerization}

For the polymerization of the contact lens materials, HEMA, NVP, MMA, EGDMA were employed as the basic combination after purification. The procedure involved washing an aqueous solution of monomer with hexanes, salting the monomer by addition of $\mathrm{NaCl}$, drying and distilling under reduced pressure. AIBN was used as the initiator. $\mathrm{ZnO}$ nanoparticles were added to the basic combination in certain proportions, and 4-vinylaniline was proportionally added to each combination to be agitated for about 30 minutes. $\mathrm{ZnO}$ nanoparticles were added to the basically combined monomers and dispersed before being polymerized in an ultrasonic benchtop cleaner (Branson 2510) for about an hour.

The polymerization of the contact lens material was conducted through thermal polymerization in $70^{\circ} \mathrm{C}$ for about 40 minutes and in $80^{\circ} \mathrm{C}$ for about 40 minutes. Lastly, it was sent for heat treatment in $100^{\circ} \mathrm{C}$ for about 40 minutes. To produce contact lenses, a cast mould- method was used. The produced contact lenses samples underwent the pre-process by being hydrated in $0.9 \%$ $\mathrm{NaCl}$ saline for 24 hours. Afterward, their physical and optical properties were measured.

The samples used in the test were named VA-1, VA-2, VA-3, VA-4, and VA-5, depending on the 4-vinylaniline content. Combinations that contained $\mathrm{ZnO}$ nanoparticles were designated by adding $\mathrm{Z}$ to their names. Table 1 summarizes the combination rates of the contact lens samples used in the test.

\subsection{Instruments and Analysis}

All contact lens samples used in the test were stored in standard saline $(0.9 \% \mathrm{NaCl}$ saline) for at least 24 hours, and then were kept at the test temperature (room temperature) for at least 2 hours to reach equilibrium before the test. A total of five samples were produced for each combination to test the individual properties, and the average values were used.

To measure and calculate the water content, the gravimetric method was implemented based on ISO 183694:2006. For the refractive index, an ABBE Refractormeter (ATAGO NAR 1T, Japan) was used based on ISO 18369-4:2006. It maintained a temperature of $20^{\circ} \mathrm{C} \pm 2^{\circ} \mathrm{C}$ and measured the samples after removing the moisture from the contact lens surfaces.

To measure wettability, the contact angle was measured by using a Phoenix-Mini from the S.E.O. For contact angle measurement, the sessile drop method was used to measure the angles of the surface of contact lens samples after dropping pure distilled water at room temperature.

Spectral transmittance was obtained by using the spectral transmittance meter (TOPCON TM-2, Japan), and

Table 1. Percent compositions of samples. Unit: \%.

\begin{tabular}{ccccccc}
\hline & HEMA & MMA & NVP & EGDMA & ${ }^{+} 4-V A$ & ZnO \\
\hline Ref. & 93.90 & 0.94 & 4.69 & 0.47 & - & - \\
VA-1 & 93.02 & 0.93 & 4.65 & 0.47 & 0.93 & - \\
VA-2 & 92.17 & 0.92 & 4.61 & 0.46 & 1.84 & - \\
VA-3 & 91.32 & 0.91 & 4.57 & 0.46 & 2.74 & - \\
VA-4 & 90.50 & 0.90 & 4.52 & 0.45 & 3.62 & - \\
VA-5 & 89.69 & 0.90 & 4.48 & 0.45 & 4.48 & - \\
\hline Ref._Z & 93.68 & 0.94 & 4.68 & 0.47 & 0.00 & 0.23 \\
VA_Z-1 & 92.81 & 0.93 & 4.64 & 0.46 & 0.93 & 0.23 \\
VA_Z-2 & 91.95 & 0.92 & 4.60 & 0.46 & 1.84 & 0.23 \\
VA_Z-3 & 91.12 & 0.91 & 4.56 & 0.46 & 2.73 & 0.23 \\
VA_Z-4 & 90.29 & 0.90 & 4.51 & 0.45 & 3.61 & 0.23 \\
VA_Z-5 & 89.49 & 0.89 & 4.47 & 0.45 & 4.47 & 0.22 \\
\hline
\end{tabular}

+4-VA: 4-vinylaniline. 
the transmittance of UV-B, UV-A and visible ray were represented in terms of percentage.

To analyze the nano-particles contained in the polymers, an atomic force microscope (XE-100, Parks System) and FE-SEM (JSM-7500F+EDS, Oxford) were used.

\section{Result and Discussion}

\subsection{Polymerization and Production}

Basic combinations of contact lenses were used in the test, and the Ref. combination produced colorless and transparent contact lenses. Since it was hydrated in standard saline for 24 hours, it showed generally flexible and soft properties. The combination with the addition of $\mathrm{ZnO}$ nanoparticles tended to result in pale white-colored transparent contact lenses. The combination to which 4-vinylaniline was added to the basic combination or the Ref. combination generated a high performance polymerthat featured a transparent brown tint. Overall, the greater the proportion of added substances, the more visible their colors were. Since all the combinations were soaked in standard saline for 24 hours, they were all flexible and soft.

After analyzing the surface conditions and the combinations with $\mathrm{ZnO}$ nanoparticles with an atomic force microscope, it was found that the particles of about 50 $70 \mathrm{~nm}$ were evenly distributed. The analysis results of the atomic force microscope on hydrogel contact lenses containing $\mathrm{ZnO}$ nanoparticles and the photos of the manufactured contact lenses' appearance and colors are shown in Figures 1-3.

\subsection{Water Content and Refractive Index}

After measuring the water content of the produced contact lenses, it was revealed that the average water content of the Ref. that did not contain 4-vinylaniline and $\mathrm{ZnO}$ nanoparticles was $38.95 \%$. This water content value is similar to normal hydrogel contact lenses. The average water contents of the VA combination to which 4-vinylaniline was added in different proportions were VA-1 (37.11\%), VA-2 (35.72\%), VA-3 (33.99\%), VA-4 (33.18\%), and VA-5 (31.10\%). Overall, greater proportions of 4-vinylaniline tended to result in less water content. It was deemed that the vinyl group that is susceptible to polymerization played a role in attaining the hydrophobic properties.

The average water content of the Ref._Z combination made by adding the Ref. combination with $\mathrm{ZnO}$ nanoparticles was measured as $38.45 \%$. This water content value is similar to normal hydrogel contact lenses, as well as the Ref. combination. The average water contents of the VA combination to which $\mathrm{ZnO}$ nanoparticles were added were VA_Z-1 (37.06\%), VA_Z-2 (34.88\%), VA_Z-3

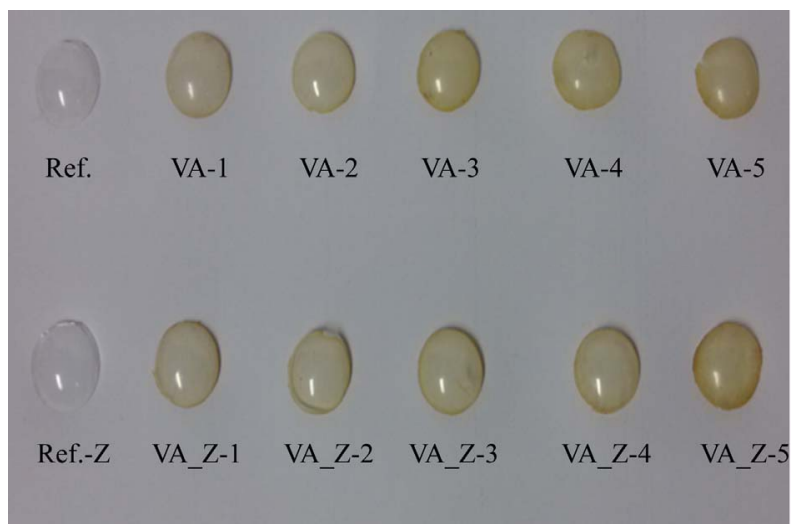

Figure 1. Photograph of tinted hydrogel lens.

(34.36\%), VA_Z-4 (32.75\%) and VA_Z-5 (30.62\%). Overall, greater proportions of 4-vinylaniline tended to result in less water content. It was found that the variation in water content induced by the addition of $\mathrm{ZnO}$ nanoparticles was not very significant. After measuring the refractive index of the produced contact lenses, the average refractive index for Ref. to which 4-vinylaniline and $\mathrm{ZnO}$ nanoparticles were not added was 1.4352 . The average refractive index of the Ref. combination to which 4-vinylaniline was added in different proportions fell into the range of 1.4472 - 1.4780. In general, greater proportions of 4-vinylaniline tended to result in greater refractive index. The average water content of the Ref._Z combination made by adding the Ref. combination with $\mathrm{ZnO}$ nanoparticle fell into the range of 1.4450 - 1.4780. Just like VA combination, greater proportions of 4-vinylaniline resulted in greater refractive index. It was found that the variation in refractive index by the addition of $\mathrm{ZnO}$ nanoparticles was not very significant. Table 2 shows the water content and refractive index of each sample.

\subsection{Wettability}

As the contact angle was measured to evaluate the wettability of the produced contact lenses, it was found that the average contact angle for the Ref. without 4-vinylaniline and $\mathrm{ZnO}$ nanoparticles was $46.96^{\circ}$. The average contact angle of the Ref._Z combination made by adding the Ref. combination with $\mathrm{ZnO}$ nanoparticles was measured as low as $42.02^{\circ}$, indicating that the addition of $\mathrm{ZnO}$ nanoparticles influenced the contact angle. The average contact angles of the VA combination to which 4-vinylaniline was added in different proportions were VA-1 $\left(54.95^{\circ}\right)$, VA-2 $\left(58.57^{\circ}\right)$, VA-3 $\left(64.68^{\circ}\right)$, VA-4 $\left(71.45^{\circ}\right)$, and VA-5 $\left(76.67^{\circ}\right)$. In general, greater proportions of 4-vinylaniline tended to result in larger contact angles, leading to a drop in wettability. This was apparently caused by the hydrophobic properties of 4-vinylaniline, which blocked the wettability on the surface.

The average contact angles of the VA_Z combination 

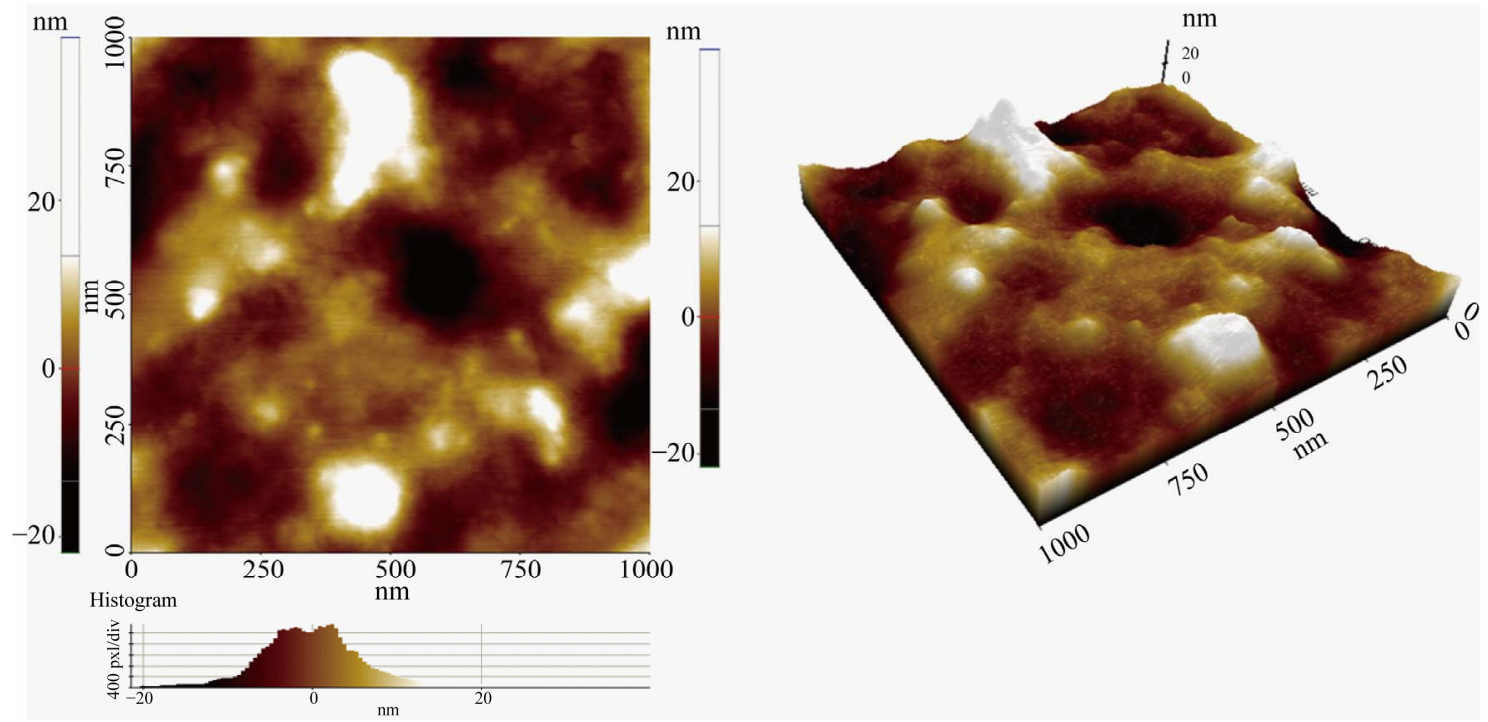

Figure 2. Surface analysis of macromolecule by AFM images (Ref._Z).

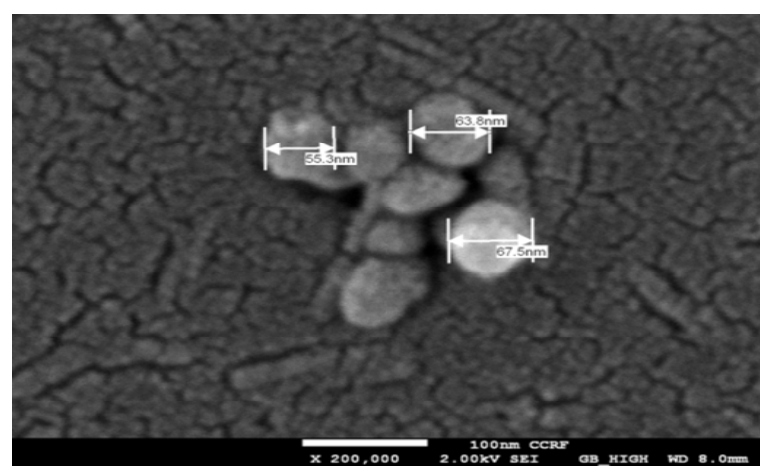

Figure 3. SEM image of contact lens sample (Ref._Z).

Table 2. It presents the water content and refractive index measurement results for each sample.

\begin{tabular}{ccc}
\hline sample & ${ }^{*} \omega_{\mathrm{H}_{2} \mathrm{O}}(\%)$ & Refractive index \\
\hline Ref. & 38.95 & 1.4352 \\
VA-1 & 37.11 & 1.4472 \\
VA-2 & 35.72 & 1.4578 \\
VA-3 & 33.99 & 1.4662 \\
VA-4 & 33.18 & 1.4704 \\
VA-5 & 31.10 & 1.4780 \\
\hline Ref._Z & 38.45 & 1.4332 \\
VA_Z-1 & 37.06 & 1.4450 \\
VA_Z-2 & 34.88 & 1.4590 \\
VA_Z-3 & 34.36 & 1.4640 \\
VA_Z-4 & 32.75 & 1.4702 \\
VA_Z-5 & 30.62 & 1.4780 \\
\hline
\end{tabular}

\footnotetext{
$\omega_{\mathrm{H}_{2} \mathrm{O}}$ is the water content.
}

made by adding 4-vinylaniline to the Ref._Z combination in different proportions were VA_Z-1 (51.18 $)$, VA_Z-2 $\left(54.37^{\circ}\right), \quad$ VA_Z-3 $\left(60.29^{\circ}\right), \quad$ VA_Z-4 $\left(73.60^{\circ}\right)$, and VA_Z-5 $\left(78.48^{\circ}\right)$. Just like VA combination, greater proportions of 4-vinylaniline tended to result in larger contact angles and decreased wettability. It was found that the addition of $\mathrm{ZnO}$ nanoparticles reduced the overall contact angle. Nevertheless, the combination with a high proportion of 4-vinylaniline did not exhibit a drop in contact angle. It can be conjectured that in a combination with a large proportion of 4-vinylaniline, wettability was influenced more by 4-vinylaniline than by $\mathrm{ZnO}$ nanoparticles. Figure 4 shows the variation in contact angle in each sample while Figure 5 represents the measurement results for contact angles.

\subsection{Spectral Transmittance}

The average transmittance was $90.06 \%$ for visible rays of the Ref. without 4-vinylaniline and $\mathrm{ZnO}$ nanoparticles. The transmittance ratio was $78.4 \%$ for UV-B and $85.0 \%$ for UV-A. In general, the transmittance in all regions was high, but it did not block ultraviolet rays.

In the VA combination in which 4-vinylaniline was added in different proportions to the Ref. combination, the visible ray transmittances were in the range of $81.6 \%$ - 87.2\%. Although, greater proportions of 4-vinylaniline tended to be accompanied by lower visible ray transmittance, it appeared that this was due to a decrease in permeability in $400-450 \mathrm{~nm}$ rather than a drop of transparency. The average UV-B transmittance ratio of the VA combination was measured as $0.4 \%-6.0 \%$, indicating that it completely blocked UV-B. For the UV-A transmittance ratio, it was measured as $12.6 \%-43.4 \%$, which meant that it blocked some UV-A. In general, it 


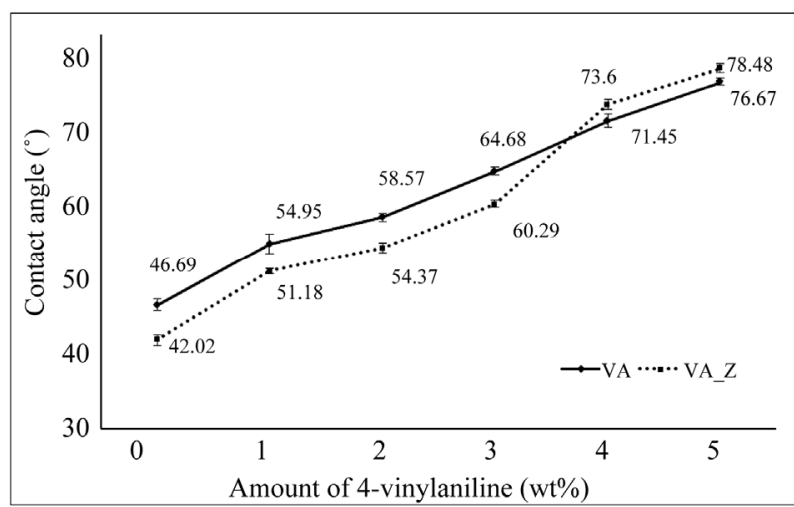

Figure 4. Effect of 4-vinylaniline on contact angle.

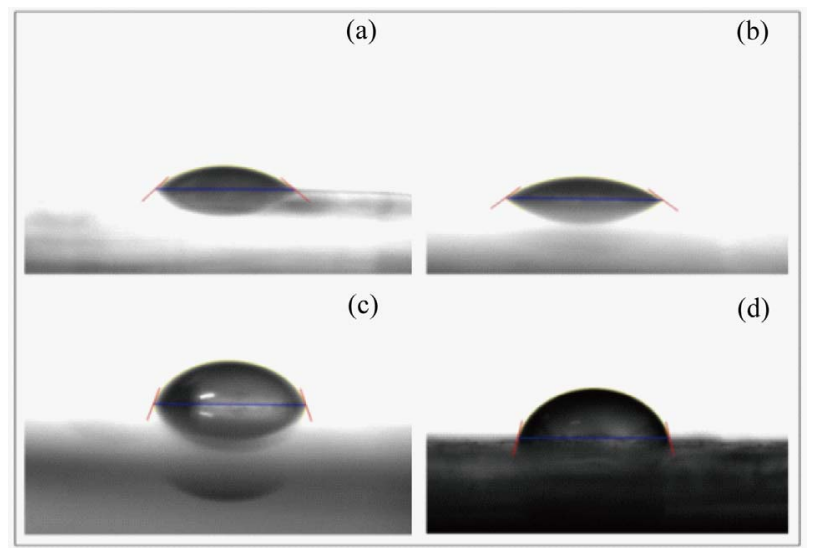

Figure 5. Contact angle photographs of contact lens samples (a) Ref.; (b) Ref._Z; (c) VA-5; (d) VA_Z-5.

was found that greater proportions of 4-vinylaniline resulted in smaller visible ray transmittance ratios. This proves that 4-vinylaniline has ultraviolet ray blocking properties. Figure 6 depicts the comparison graphs for the Ref. combination and VA-5 combination.

In the Ref._Z combination in which $\mathrm{ZnO}$ nanoparticles were added to the Ref. combination, the transmittance ratio was $83.4 \%$ for visible rays. The transmittance ratio was $40.0 \%$ for UV-B and $45.8 \%$ for UV-A. In general, the visible ray transmittance in comparison to the Ref. combination fell in all regions, especially in the ultraviolet ray region. In case of the $\mathrm{ZnO}$ nanoparticles, it was found that it has some ultraviolet blocking ability. Figure 7 represents the spectral transmittance comparison graphs of the Ref. combination and Ref.-Z combination.

In the VA_Z combination made by adding 4-vinylaniline to the Ref._Z combination in different proportions, it was revealed that the visible ray transmittance was in the range of $71.8 \%-77.8 \%$. Overall, greater proportions of 4-vinylaniline tended to result in smaller visible ray transmittance. The UV-B transmittance was measured as $0.2 \%-2.0 \%$, signifying complete UV blocking, whereas that of UV-A was from $5.8 \%$ to $17.6 \%$, which shows a significant ability to block UV-A. The VA_Z

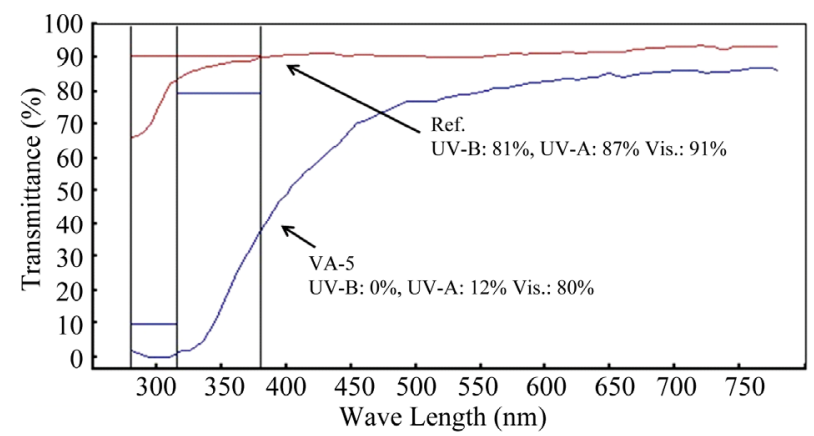

Figure 6. Spectral transmittances of samples (Ref. and VA-5).

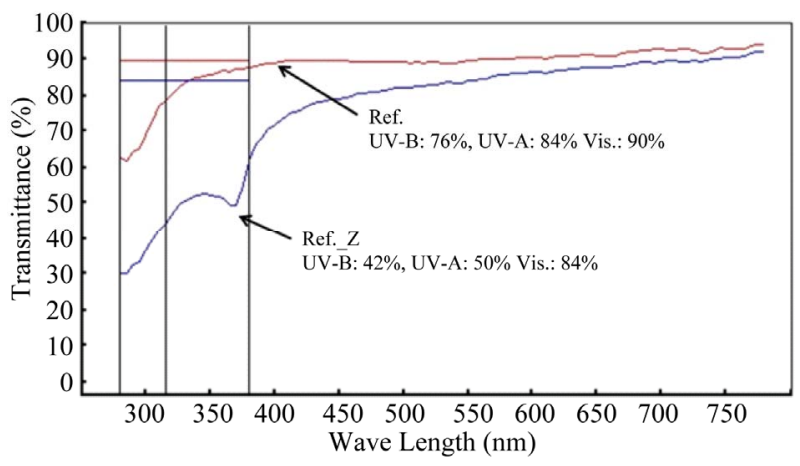

Figure 7. Spectral transmittances of samples (Ref. and Ref._Z).

combination, compared to the VA combination to which $\mathrm{ZnO}$ nanoparticles were not added, had a sizably low spectral transmittance for UV-A, which seemed to be attributable to the influence of the $\mathrm{ZnO}$ nanoparticle. Furthermore, the visible ray transmittance was also low, which could be due to the overall decrease in transmittance, rather than a specific region. Figure 8 presents the spectral transmittance comparison graphs for the VA-3 combination and VA_Z-3 combination. The spectral transmittance results in all combinations are shown in Table 3.

\section{Conclusions}

In this research, the hydrogel contact lenses materials that contain $\mathrm{ZnO}$ nanoparticles with luminescence property in the ultraviolet region were prepared and their physical and optical properties were investigated. 4-Vinlyaniline which is used as an ingredient of dyes and $\mathrm{ZnO}$ nanoparticles was added in different proportions to analyze the variations in their physical and optical properties.

The color of the produced high performance polymers generally ranged from pale gray to yellowish brown due to the $\mathrm{ZnO}$ nanoparticles and 4-vinlyaniline and their color concentrations differed depending on the added proportions. Larger proportions of 4-vinlyaniline showed a decrease in water content but an increase in refractive 


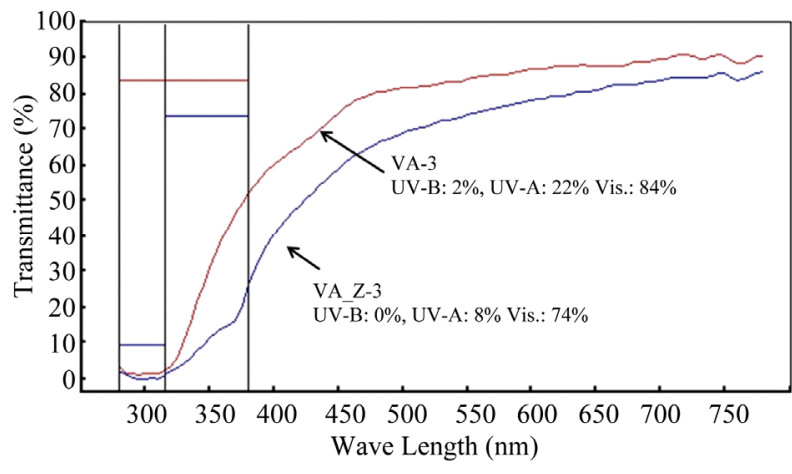

Figure 8. Spectral transmittances of samples (VA-3 and VA_Z-3).

Table 3. Spectral transmittances of samples.

\begin{tabular}{cccc}
\hline sample & UV-B & UV-A & Vis. \\
\hline Ref. & 78.4 & 85.0 & 90.6 \\
VA-1 & 6.0 & 43.4 & 87.2 \\
VA-2 & 2.0 & 28.6 & 85.4 \\
VA-3 & 1.0 & 22.8 & 85.2 \\
VA-4 & 1.0 & 21.4 & 83.6 \\
VA-5 & 0.4 & 12.6 & 81.6 \\
\hline Ref._Z & 40.0 & 45.8 & 83.4 \\
VA_Z-1 & 2.0 & 17.6 & 77.8 \\
VA_Z-2 & 0.6 & 11.0 & 74.0 \\
VA_Z-3 & 0.4 & 11.0 & 76.8 \\
VA_Z-4 & 0.2 & 6.8 & 73.8 \\
VA_Z-5 & 0.2 & 5.8 & 71.8 \\
\hline
\end{tabular}

index. And it was uncovered that the addition of $\mathrm{ZnO}$ nanoparticles played a negligible role. The wettability of contact lens tended to diminish with the increase in the proportion of 4-vinlyaniline. The addition of $\mathrm{ZnO}$ nanoparticle was also shown to exert some influence over contact angle.

In terms of spectral transmittance, the addition of $\mathrm{ZnO}$ nanoparticles led to the decreasing in transmittance ratio in the ultraviolet region. If 4-vinlyaniline was added, it also reduced the transmittance ratio in the ultraviolet region.

It is expected that the results would contribute to a wider application of contact lenses containing $\mathrm{ZnO}$ nanoparticles which are to be used as ultraviolet ray blocking contact lenses, and aniline-group materials can be employed to show pale colors in hydrophilic lenses and utilized as an ultraviolet ray blocking contact lens material.

\section{REFERENCES}

[1] T. H. Kim, K. H. Ye and A. Y. Sung, "UV-Blocking Effect of Ophthalmic Material Containing Benzophenone,”
Journal of the Korean Chemical Society, Vol. 53, No. 3, 2009, pp. 391-398.

http://dx.doi.org/10.5012/jkcs.2009.53.3.391

[2] K. Diduch, R. Wubbenhorst and S. Kucharski, "Photocurrent Generation of Bi-Functional Carbazole Containing Polymers,” Synthetic Metals, Vol. 139, No. 2, 2003, pp. 515-520. http://dx.doi.org/10.1016/S0379-6779(03)00352-7

[3] V. A. Soloukhin, W. Postumus, J. C. M. Brokken-Zijp and J. Loos, "Mechanical Properties of Silica-(Meth)Acrylate Hybrid Coatings on Polycarbonate Substrate," Polymer, Vol. 43, No. 23, 2002, pp. 6169-6181. http://dx.doi.org/10.1016/S0032-3861(02)00542-6

[4] T. H. Kim and A. Y. Sung, "Copolymerization and Contact Lens Application of HEMA-Substituted Polyphosphazene," Journal of the Korean Chemical Society, Vol. 53, No. 3, 2009, pp. 340-344.

http://dx.doi.org/10.5012/jkcs.2009.53.3.340

[5] T. H. Kim, K. H. Ye, Y. S. Kwon and A. Y. Sung, "Study on the Silicone Contact Lens Using AA and BMA,” Journal of the Korean Ophthalmic Optics Society, Vol. 11, No. 3, 2006. pp. 259-265.

[6] K. Vimala, M. M. Yallapu, K. Varaprasad, N. Narayana Reddy, S. Ravindra, N. S. Naidu and K. MohanaRaju, "Fabrication of Curcumin Encapsulated Chitosan-PVA Silver Nanocomposite Films for Improved Antimicrobial Activity" Journal of Biomaterials and Nanobiotechnology, Vol. 2, No. 1, 2011, pp. 55-64. http://dx.doi.org/10.4236/jbnb.2011.21008

[7] K. H. Ye, S. H. Choi and A. Y. Sung, "Study on the Antibiosis and Physical Properties of Copolymer Containing Silver," Journal of the Korean Chemical Society, Vol. 53, No. 3, 2009, pp. 562-546.

[8] K. H. Ye and A. Y. Sung, "Synthesis of Polymer Materials Containing Platinum Nanoparticles and Their Application for Contact Lenses,” Journal of the Korean Chemical Society, Vol. 54, No. 1, 2010, pp. 99-104. http://dx.doi.org/10.5012/jkcs.2010.54.01.099

[9] K. H. Ye and A. Y. Sung, "Study on the Synthesis and Physical Properties of Ophthalmic Polymer Containing Gold Nanoparticles,” Journal of the Korean Chemical Society, Vol. 54, No. 2, 2010, pp. 222-227. http://dx.doi.org/10.5012/jkcs.2010.54.02.222

[10] R. W. Young, "Solar Radiation and Age-Related Macular Degeneration,” Survey of Ophthalmology, Vol. 32, No. 4, 1998, pp. 252-269. http://dx.doi.org/10.1016/0039-6257(88)90174-9

[11] W. S. Duke-Elder and P. M. Duke-Elder, “A Histological Study on the Action of Short-Waved Light upon the Eye, with a Note on 'Inclusion Bodies'," British Journal of Ophthalmology, Vol. 13, No. 1, 1929, pp. 1-37. http://dx.doi.org/10.1136/bjo.13.1.1

[12] S. Zigman, T. Yulo and J. Schultz, "Cataract Induction in Mice Exposed to near UV Light,” Ophthalmic Research, Vol. 6, No. 2-4, 1974, pp. 259-270. http://dx.doi.org/10.1159/000264710

[13] M. Kennedy, K. H. Kim, B. Harten, et al., "Ultraviolet Irradiation Induces the Production of Multiple Cytokines by Human Corneal Cells,” Investigative Ophthalmology 
\& Visual Science, Vol. 38, No. 12, 1997, pp. 2483-2491.

[14] A. Podskochy, L. Gan and P. Fagerholm, "Apoptosis in UV-Exposed Rabbit Corneas,” Cornea, Vol. 19, No. 1, 2000, pp. 99-103.

http://dx.doi.org/10.1097/00003226-200001000-00019

[15] C. X. Cai, D. E. Birk and T. F. Linsenmayer, "Nuclear Ferritin Protects DNA from UV Damage in Corneal Epithelial Cells,” Molecular Biology of the Cell, Vol. 9, No. 5, 1998, pp. 1037-1051. http://dx.doi.org/10.1091/mbc.9.5.1037

[16] T. H. Kim and A. Y. Sung, "Study on the High-Performance Lens Material Containing 4-Vinylpyridine,” Jour- nal of the Korean Chemical Society, Vol. 54, No. 4, 2010, pp. 487-490.

[17] T. H. Kim and A. Y. Sung, "Study on the Opthalmic Lens Materials with High Refractive Index Containing Vinylanisole,” Journal of the Korean Chemical Society, Vol. 54, No. 6, 2010, pp. 755-760.

http://dx.doi.org/10.5012/jkcs.2010.54.6.755

[18] T. H. Kim and A. Y. Sung, "Study on the Ophthalmic Optical Application of Poly(HEMA-co-FMA) Containing 2,3-Naphthalocyanine Bis(trihexylsilyloxide), Journal of the Korean Chemical Society, Vol. 55, No. 4, 2011, pp. 723-728. http://dx.doi.org/10.5012/jkcs.2011.55.4.723 